\title{
Efficacy and safety of thrice weekly DOTS in tuberculosis patients with and without HIV co-infection: an observational study
}

Richa Vashishtha', Krishna Mohan', Bhagteshwar Singh', Satish K Devarapu', Vishnubhatla Sreenivas², Sanjay Ranjan', Deepak Gupta', Sanjeev Sinha ${ }^{1}$ and Surendra K Sharma ${ }^{1 *}$

\begin{abstract}
Background: Despite the latest World Health Organization guidelines advocating daily therapy in HIV-TB co-infected individuals, there are few recent studies comparing outcomes of thrice-weekly anti-tuberculosis treatment in HIV-positive and HIV-negative patients with TB. The present study sets out to compare TB treatment outcomes in these two groups in the Indian national programme, which currently involves thrice-weekly therapy for all, regardless of HIV status.

Methods: HIV-positive and HIV-negative were consecutively screened for enrolment into this prospective observational study, carried out at the All India Institute of Medical Sciences hospital, New Delhi, India, between 2006 and 2010. Patients were given short-course thrice-weekly rifampicin-based therapy, with all HIV-positive patients being started on highly active antiretroviral therapy at least 14 days after commencing TB treatment. Patients were regularly followed-up for 24 months after completion of treatment.

Results: 150 HIV-positive, 155 HIV-negative patients were enrolled consecutively for the study. Significantly higher treatment success $(93.5 \%$ vs. $76.7 \%$ at end of treatment, $p<0.001)$ and lower mortality $(2.8 \%$ vs. $21.6 \%$ on follow up, $p<0.001$ ) were observed in HIV-negative patients. No significant difference was found in treatment failure $(p=0.16)$, sputum smear $(p=0.58)$ and culture conversion $(p=0.55)$, and non-serious adverse event incidence $(p=0.851)$ between the two groups. Low baseline CD4 cell count $\left(<100\right.$ cells $\left./ \mathrm{mm}^{3}\right)$ was the only predictor of mortality in HIV-TB patients (odds ratio $8 \cdot 43, p=0 \cdot 013$ ).
\end{abstract}

Conclusions: Thrice-weekly anti-tuberculosis therapy is more effective in HIV-negative than in HIV-positive patients. However, outcomes in this HIV co-infected cohort were found to be similar to those reported previously with daily therapy, with no safety concerns. This should prompt further study into whether intermittent or daily therapy should be used universally in resource-poor settings, using large well executed randomised controlled trials.

Trial registration: NCT No. 00698334

Keywords: Antitubercular agents/therapeutic use, Tuberculosis/drug therapy, Mortality, HIV infections/complications, Treatment outcome

\footnotetext{
*Correspondence: sksharma.aiims@gmail.com

${ }^{1}$ Department of Medicine, All India Institute of Medical Sciences, Ansari Nagar, New Delhi 110029, India

Full list of author information is available at the end of the article
} 


\section{Background}

Tuberculosis (TB) and human immunodeficiency virus (HIV) infection are leading causes of morbidity and mortality worldwide [1,2]. Despite significant progress in TB control in recent years [3], the HIV epidemic has posed a substantial challenge in the form of HIV and TB coinfection [3-5]. There is much debate over how antituberculosis therapy (ATT) should be administered to such individuals, given their immunocompromised state, potential for drug-drug interactions [6,7], higher mortality rates [1-4,8-10] and differences in presentation and organ involvement $[3,6,7]$. Some areas are widely agreed upon, such as modification of antiretroviral therapy (ART) to avoid interactions $[3,4,6,7]$, ATT commencing before ART in treatment-naïve patients $[3,6]$, and avoidance of once- or twice-weekly dosing schedules [2,3,6,11]. However, specific issues which are being increasingly debated and researched include optimal duration of therapy [3,6-8,11-15], use of daily versus intermittent regimens (WHO recommend daily in at least the intensive phase) $[3,15,16]$, and optimal timing of ART commencement [2,3,5-7].

A recent meta-analysis by Khan and colleagues suggests that there is a relative lack of high quality data on outcomes of ATT in HIV-TB co-infected patients, especially with regards to intermittent dosing schedules [16]. When comparing daily and thrice-weekly therapy, the comparative meta-analysis included over 3000 patients who had received daily treatment in the intensive phase, but only 211 who were treated with thrice-weekly regimens [16]. There are many studies reporting outcomes of ATT in HIV-infected patients with sputum smear and culture positive pulmonary TB [2,10,13-15,17-19] but few including smear-negative and extrapulmonary TB $[8,9,11,12]$. In the real-world, co-infected individuals are much more likely to have these challenging forms of $\mathrm{TB}$ $[1,3]$, thus highlighting lacunae in research.

It is our personal observation that $\mathrm{TB}$ treatment using the current Indian national guidelines, which, in line with the previous WHO guidance, advocate 6-month thrice-weekly ATT irrespective of HIV status, outcomes are poorer in HIV-infected individuals. Hence, an observational study was undertaken to compare outcomes of current TB treatment in ATT- and ART-naïve HIVpositive and HIV-negative patients.

\section{Methods}

\section{Study design and case selection}

This prospective observational study was conducted at the All India Institute of Medical Sciences hospital, a large tertiary centre in New Delhi, northern India. The Department of Medicine provides specialist TB and HIV services to Delhi and neighbouring states. All socioeconomic strata are represented, but the majority are in lower groups [20].
ATT and ART naïve patients aged 18-65 years receiving a new diagnosis of active TB between April 2006 and October 2010, were consecutively assessed for enrolment. The following exclusion criteria were applied: patients with previous history of TB, ATT or ART; pregnant, lactating and seriously unwell patients; those with significantly raised serum transaminase levels or concomitant renal impairment, liver failure, diabetes mellitus, epilepsy or alcoholism; patients with hepatitis B or C virus coinfection; those unable to attend follow-up; and any patient having been on ATT for more than two weeks during the current schedule.

Two groups were formed: one with ART-naive patients having a new or existing diagnosis of concurrent HIV infection, and HIV-negative patients in the other. The All India Institute of Medical Sciences Institute Ethics Committee approved the study, and written informed consent was obtained from patients.

\section{Diagnosis and baseline assessment}

All patients were counselled and tested for HIV using a licensed third generation ELISA kit as described previously [20]. TB was diagnosed using investigations as indicated and possible. These included Ziehl Neelson smear microscopy followed by Lowenstein Jensen culture of sputum and any body fluids, secretions, tissue or pus; Mycobacterium tuberculosis polymerase chain reaction (PCR); histo- and cytopathological examination of tissue or fluid (including broncho-alveolar lavage if required); and various imaging modalities. For example, all subjects with suspected pulmonary $\mathrm{TB}$ underwent sputum or broncho-alveolar lavage smear and culture, whereas if fluids such as cerebrospinal fluid and pleural aspirate were smear negative, then $M$. tuberculosis PCR was performed.

TB diagnosis in suspected cases was classified as definitive (diagnosed by smear, culture or PCR) or probable (clinical and radiological response to treatment and suggestive radiology, histology, cytology or fluid biochemistry) [21].

All patients underwent baseline clinical assessment, chest radiography and blood tests, including CD4 lymphocyte count by flow cytometry (Becton Dickinson, USA). Plasma viral load estimation was performed in HIV-infected individuals, using either semi-quantitative (Amplicor HIV-1 Monitor Test, Roche, Indianapolis) or real time PCR methods. In pulmonary TB, chest radiographic severity was graded [22]. In smear-positive patients, the sputum smear bacillary load was graded as scanty, $1+, 2+$ or $3+$ as per WHO guidelines [23].

\section{Treatment}

Thrice-weekly rifampicin-based directly observed ATT was given as per the Indian Revised National TB Control 
Programme guidelines [24]. This involved two months of thrice weekly isoniazid (600 mg), rifampicin (450 mg; 600 $\mathrm{mg}$ if weight $>60 \mathrm{~kg})$, pyrazinamide $(1500 \mathrm{mg})$ and ethambutol (1200 mg) [intensive phase], and four months of thrice weekly isoniazid and rifampicin (doses as above) [continuation phase] [24].

Extension of ATT was considered in patients who showed clinical and/or radiological signs of disease progression or partial response, or persisting sputum smear positivity at the end of the intensive phase, after a consensus decision by two or more investigators. The extensions were of one, two or three months, of either the intensive (one month) or continuation phase (maximum three months). If despite extension, a patient was still deemed to have incomplete response at the end of treatment, this was labelled as treatment failure. They were started on 'category II' ATT as per the national guidelines (i.e. longer therapy with streptomycin added) [24], subsequently guided by susceptibilities from susceptibility testing results.

All HIV-positive patients received free highly active ART as per India's National AIDS Control Organisation guidelines: lamivudine (150 $\mathrm{mg} \mathrm{q} 12 \mathrm{~h})$ and efavirenz $(600 \mathrm{mg}$ qd) with either zidovudine $(300 \mathrm{mg}$ $\mathrm{q} 12 \mathrm{~h}$, in those with haemoglobin $>8 \mathrm{~g} / \mathrm{dl}$ ) or stavudine (30 $\mathrm{mg} \mathrm{q} 12 \mathrm{~h}$, if $\mathrm{Hb}<8 \mathrm{~g} / \mathrm{dl}$ ), which was started two weeks or more after commencement of, but during, ATT [25]. After completion of ATT, efavirenz was replaced with nevirapine (200 mg q12h) [25]. All HIVinfected patients diagnosed in 2008 or later were started on prophylactic co-trimoxazole therapy as per changes in national guidelines [25]. Of those diagnosed before 2008, all with CD4 count $<200$ cells $/ \mathrm{mm}^{3}$ received prophylaxis.

\section{Follow-up}

Follow-up was fortnightly during the first two months of treatment, subsequently monthly up to the end of ATT, then at $3,6,12,18$ and 24 months post-ATT. Follow-up, using a structured pro forma document, included clinical assessment, monitoring for opportunistic infections (in HIV-positive patients), routine blood investigations, sputum smear and culture if appropriate and possible, and repeat imaging as at enrolment to monitor response to treatment.

In pulmonary $\mathrm{TB}$ patients, monthly chest radiographs and fortnightly sputum smear and culture were performed.

CD4 cell counts were measured at enrolment and 6 months after starting treatment in all, with subsequent six-monthly counts in HIV-positive patients only. Plasma viral loads were measured at baseline, 6 months after commencing ATT, and then after a further 6 and 24 months in the HIV-positive group.

Adherence to treatment was checked at each visit to the DOTS (thrice weekly in the intensive phase, weekly thereafter) or ART centre (monthly) by questioning and blister pack checks. These were supported by a medical social worker.

Adverse events observed were noted and communicated to the principal investigator and ethics committee. These were classified as non-serious and serious adverse events. The former included mild and moderate events such as nausea, abdominal pain and itch; whereas immune reconstitution inflammatory syndrome, new onset anaemia, deaths and other severe or life-threatening events were labelled as serious.

Mycobacterium tuberculosis genotyping was performed with a spoligotyping commercial kit (Ocimum Biosolutions Ltd., Hyderabad, India) according to a previously described method, on relapse samples and corresponding baseline samples, to differentiate exogenous reinfection from endogenous reactivation [26].

\section{Endpoints}

The primary endpoint was outcome at the end of initial treatment, as defined by the WHO: cure (smear- and culture-negativity at end of treatment in smear-positive pulmonary $\mathrm{TB}$ ), treatment completed (no evidence of treatment failure or cure), treatment failure (smear- or culture-positivity in the last 2 months of treatment, or evidence of clinical and/or radiological worsening or partial response), or death [3]. The term 'treatment success' refers to the sum of 'cure' and 'treatment completed', and was used as the main comparative outcome, due to the inclusion of smear-negative and extrapulmonary cases in whom the 'cure' label cannot be applied.

Secondary endpoints were treatment outcome at the end of 24-month follow-up; duration of initial treatment; sputum smear- and culture-conversion rate in sputumpositive pulmonary TB patients; adverse events; and mortality in HIV-co-infected patients.

\section{Statistical analysis}

Sample size calculation was as follows: an initial sample of 165 patients would be required in each group would be sufficient to detect a difference in treatment success of $15 \%$ (HIV negative $=85 \%$, HIV positive $=70 \%$, with two sided $5 \%$ significance level and $90 \%$ power), assuming $25 \%$ attrition. Primary analysis was by modified intention to treat, with on-treatment analysis for adverse events. All patients receiving at least one dose of ATT were included for treatment outcome analysis. Comparisons between groups were analysed using Fisher's exact or Pearson chi-squared tests for categorical data, and unpaired t-test or Wilcoxon Rank-sum test for qualitative characteristics. Odds ratios were calculated for independent risk factors for mortality in the HIV-positive group. All analysis was carried out using Stata ${ }^{\circ}$ version 11. 


\section{Results}

\section{Patients}

305 patients were enrolled from the 497 consecutively screened for eligibility; 155 were HIV-negative and 150 tested positive for HIV (Figure 1). End of treatment outcomes underwent modified intention to treat analysis: all patients undergoing any treatment were included. Follow-up based outcomes, also undergoing modified intention to treat analysis, included all except those still under regular follow-up $(n=211)$. In the context of adverse events, on treatment analysis was applied to all those receiving any ATT $(n=305)$.

Baseline characteristics for the two groups are compared in Table 1. Body mass index and serum bilirubin were not found to differ significantly. Foci of TB involvement in each group are shown in Table 2. Extrapulmonary involvement $(\mathrm{p}=0.001)$ and dissemination $(\mathrm{p}<0.001)$ were significantly more common in patients with HIV. For pulmonary TB patients, Tables 3 depicts the baseline sputum smear results and chest radiograph severity. The bacillary load was significantly higher in HIV-negative than HIV-positive smear-positive patients $(\mathrm{P}<0 \cdot 001)$.

The basis of diagnosis for each group is shown in Table 4. Using the classification described above, 74 (49.3\%) of the HIV-positive and $121(78 \cdot 1 \%)$ of HIVnegative diagnoses were 'definitive'. Of the 83 (27.2\%) 'radio-clinical' cases (diagnosed on the basis of radiological findings and clinical features), all but 11 of these displayed full response to treatment - of those who did not, six died, four were lost to follow-up during treatment, and one failed treatment. Extensive attempts were made to rule out other diagnoses.

\section{End of treatment outcome}

Treatment outcome at the end of ATT for each group is detailed in Table 5. Treatment success was achieved in $76.7 \%$ of the HIV-positive and $93.5 \%$ of the HIVnegative patients $(\mathrm{p}<0 \cdot 001)$. There were nine HIVpositive treatment failures; four in the HIV-negative group ( $p=0 \cdot 16)$, of which six (two pulmonary) and four (three pulmonary) were microbiologically confirmed, respectively. One HIV-infected patient who failed initial ATT, was well after re-treatment with a 'category II' regimen, but later relapsed, and after the end of scheduled follow-up was diagnosed with multi-drug resistant (MDR) pulmonary TB. One HIV-negative patient failed the re-treatment regimen and was subsequently diagnosed as having MDR pulmonary TB.

Thirteen HIV-positive and two HIV-negative patients died while on treatment $(\mathrm{p}<0 \cdot 01)$. Thirteen (four defaulters, nine untraceable) HIV-positive patients were lost to followup; as were four HIV-negative patients (all untraceable). One patient with HIV had to have their treatment modified permanently due to isoniazid-induced psychosis.

A total of $35(23 \cdot 3 \%) \mathrm{HIV}$-positive patients required extensions of ATT, whereas only $13(7 \cdot 8 \%)$ of the HIVnegative patients had their ATT extended $(p=0 \cdot 001)$. Each group had a median additional duration of 1 month.

Comparison of baseline BMI and laboratory parameters with those at 6 months of treatment is provided in Table 6. Serum albumin and CD4 counts increased significantly in HIV-positive patients.

Subgroup analysis was performed on initial treatment outcomes of sputum smear-positive pulmonary TB patients with and without HIV co-infection, who had completed ATT (Table 7). One hundred of 105 patients

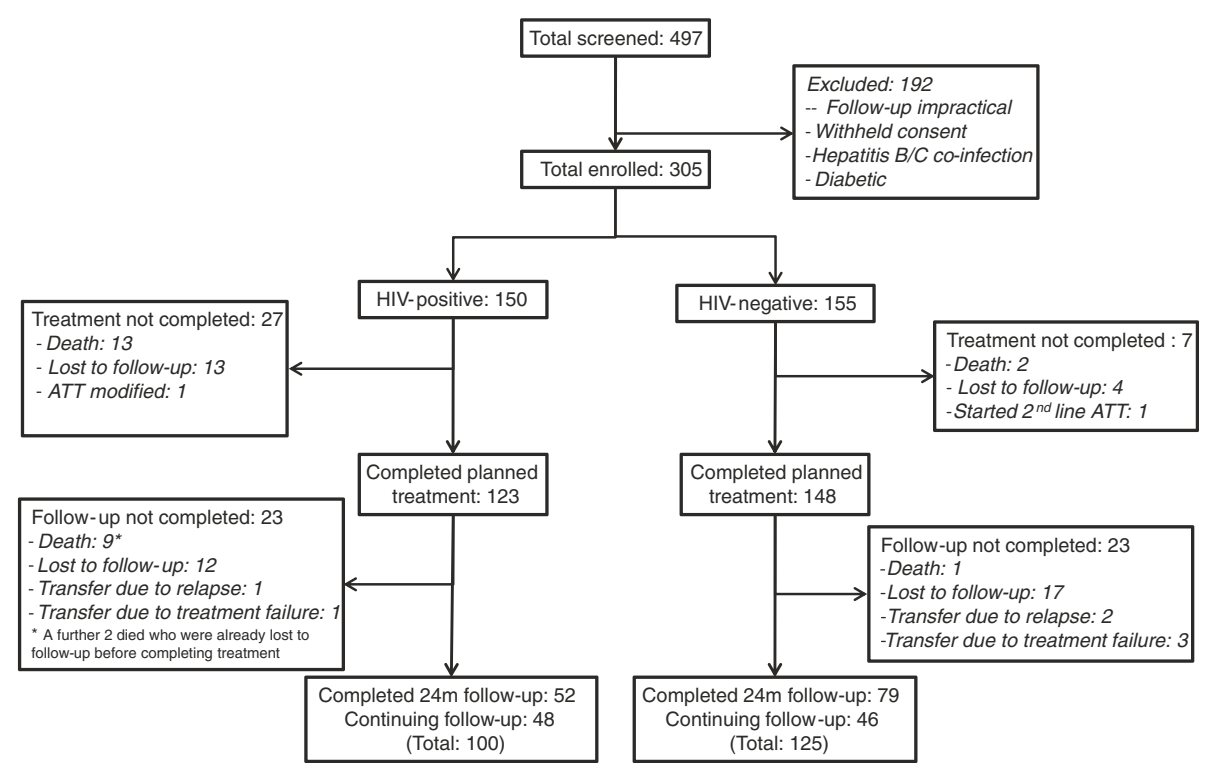

Figure 1 Study profile. 
Table 1 Baseline characteristics

\begin{tabular}{lccc}
\hline Characteristic & $\begin{array}{c}\text { HIV }+ \\
(\mathbf{n}=\mathbf{1 5 0})\end{array}$ & $\begin{array}{c}\text { HIV- } \\
(\mathbf{n}=\mathbf{1 5 5})\end{array}$ & P-value \\
\hline Age & $34.2 \pm 8.3$ & $27.2 \pm 9.5$ & $<0.001$ \\
Gender (M:F, \%) & $83: 17$ & $58: 42$ & $<0.001$ \\
BMI (kg/m²) & $18.1 \pm 2.8$ & $17.9 \pm 3.6$ & 0.48 \\
Haemoglobin (g/dL) & $10.0 \pm 2.2$ & $11.1 \pm 1.8$ & $<0.001$ \\
Erythrocyte sedimentation & $49.4 \pm 23.6$ & $43.7 \pm 21.4$ & 0.03 \\
rate (mm/hr) & & & \\
Absolute lymphocyte & $2071 \pm 1010$ & $2435 \pm 938$ & 0.001 \\
count (/mm ${ }^{3}$ ) & & & \\
CD4 count (/mm ${ }^{3}$ ) & $194 \pm 167$ & $593 \pm 232$ & $<0.001$ \\
Albumin (g/dL) & $3.5 \pm 0.7$ & $4.2 \pm 0.6$ & $<0.001$ \\
$\begin{array}{l}\text { Bilirubin (mg/dL) } \\
\text { Alanine aminotransferase }\end{array}$ & $0.65 \pm 0.24$ & $0.67 \pm 0.16$ & 0.48 \\
(IU/L) & $53.7 \pm 42.5$ & $39.2 \pm 21.9$ & $<0.001$ \\
$\begin{array}{l}\text { Aspartate aminotransferase } \\
\text { (IU/L) }\end{array}$ & $48.2 \pm 70.3$ & $32.7 \pm 21.0$ & $<0.01$ \\
$\begin{array}{l}\text { Uric acid (units) } \\
\text { Viral load (log copies/mL) }\end{array}$ & $5.23(4.74-5.76)$ & & \\
\hline Mean +/- standard deviation except median (inter-quartile range) in viral load. & & &
\end{tabular}

(95 - 2\%) in the HIV-negative group and 36 of 52 (69.2\%) HIV-positive patients had treatment success $(\mathrm{p}<0 \cdot 001)$.

Sputum smear (32/38 [84.2\%] HIV+, 64/80 [80.0\%] HIV-, $\mathrm{p}=0.58)$ and culture (29/34 [85.3\%] HIV+, 71/88 [80.7\%] HIV-, $\mathrm{p}=0.55$ ) conversion proportions in sputum-positive pulmonary patients at two months (up to 9 weeks) were found to be similar among the two groups.

\section{Follow-up}

At the time of analysis, a total of $123 / 150$ (82\%) of the HIV-positive and 148/155 (95.5\%) HIV-negative

Table 2 Systemic foci of TB involvement

\begin{tabular}{lccc}
\hline & $\begin{array}{c}\text { HIV }+ \\
(\mathbf{n}=\mathbf{1 5 0 )}\end{array}$ & $\begin{array}{c}\text { HIV- } \\
(\mathbf{n}=\mathbf{1 5 5})\end{array}$ & $\begin{array}{c}\text { Overall } \\
(\mathbf{n}=\mathbf{3 0 5})\end{array}$ \\
\hline Pulmonary TB & $\mathbf{8 9 ( 5 9 . 3 \% )}$ & $\mathbf{1 1 9}(\mathbf{7 6 . 8 \% )}$ & $\mathbf{2 0 8}(\mathbf{6 8 . 2 \% )}$ \\
Pulmonary only & $49(32.7 \%)$ & $111(71.6 \%)$ & $160(52.5 \%)$ \\
Miliary & $2(1.3 \%)$ & $2(1.3 \%)$ & $4(1.3 \%)$ \\
Disseminated* & $38(25.3 \%)$ & $6(3.9 \%)$ & $44(14.4 \%)$ \\
Extrapulmonary TB & $\mathbf{6 1 ( 4 0 . 7 \% )}$ & $\mathbf{3 6}(\mathbf{2 3 . 2} \%)$ & $\mathbf{9 7}(\mathbf{3 1 . 8 \% )}$ \\
Lymph node & $35(23.3 \%)$ & $23(14.8 \%)$ & $58(19.0 \%)$ \\
Pleural & $5(3.3 \%)$ & $4(2.6 \%)$ & $9(3.0 \%)$ \\
Intestinal & 0 & $1(0.6 \%)$ & $1(0.3 \%)$ \\
Genitourinary & 0 & $1(0.6 \%)$ & $1(0.3 \%)$ \\
Peritoneal (ascites) & 0 & $1(0.6 \%)$ & $1(0.3 \%)$ \\
Disseminated & $21(14 \%)$ & $6(3.9 \%)$ & $27(8.9 \%)$ \\
\hline
\end{tabular}

* Dissemination was defined as involvement of two or more non-contiguous organ sites [27]. Patients were labelled as 'pulmonary, disseminated' in case of presence of concomitant pulmonary and distant extrapulmonary disease, or 'extrapulmonary, disseminated' if there was evidence of dissemination without any lung parenchymal involvement.
Table 3 Baseline sputum smear and culture results for pulmonary TB patients

\begin{tabular}{lccc}
\hline $\mathbf{n}$ (\%) & $\begin{array}{c}\text { HIV + } \\
(\mathbf{n}=\mathbf{8 9})\end{array}$ & $\begin{array}{c}\text { HIV- } \\
(\mathbf{n}=\mathbf{1 1 9})\end{array}$ & $\begin{array}{c}\text { Overall } \\
(\mathbf{n}=\mathbf{2 0 8})\end{array}$ \\
\hline Smear results & & & \\
Negative & $24(27.0 \%)$ & $9(7.6 \%)$ & $33(15.9 \%)$ \\
Unable to produce sputum & $19(21.3 \%)$ & $7(5.9 \%)$ & $26(12.5 \%)$ \\
Total positive & $46(51.6 \%)$ & $103(86.6 \%)$ & $149(71.6 \%)$ \\
Scanty & $8(17.4 \%)$ & $4(3.9 \%)$ & $12(8.1 \%)$ \\
$1+$ & $22(47.8 \%)$ & $24(23.3 \%)$ & $46(30.9 \%)$ \\
$2+$ & $6(13.0 \%)$ & $25(24.3 \%)$ & $31(20.1 \%)$ \\
$3+$ & $8(17.4 \%)$ & $50(48.5 \%)$ & $58(38.9 \%)$ \\
Grading unavailable (Positive) & $2(4.3 \%)$ & 0 & $2(1.3 \%)$ \\
Culture results & & & \\
Negative & $24(27.0 \%)$ & $5(4.2 \%)$ & $29(13.9 \%)$ \\
Unavailable & $24(27.0 \%)$ & $10(8.4 \%)$ & $34(16.3 \%)$ \\
Positive & $41(46.1 \%)^{*}$ & $104(87.4 \%)^{*}$ & $145(69.7 \%)$
\end{tabular}

\section{Chest radiograph severity $\dagger$}

No infiltrates

$10(11.2) \quad 2(1.7) \quad 12(5.8)$

Minimal lesions

$25(28.1) \quad 27(22.7)$

$52(25.0)$

Moderately advanced

$38(42.7)$

$61(51.3)$

$99(47.6)$

Far advanced

$16(18.0)$

$29(24.4)$

$45(21.6)$

作 HIV-positive patients were smear-positive but culture negative, and one HIV-positive individual was smear-positive, but their culture result was not available.

+ This includes those unable to produce sputum despite sputum induction (see 'smear results' section of Table 3), as well as those whose sputum culture results were not available for other reasons.

† According to the National Tuberculosis Association of the USA classification [22].

patients had completed planned treatment of 6 to 9 months (Figure 1).

Table 8 shows treatment outcome at 24 months followup (i.e. excluding patients under active follow-up, but including deaths and loss to follow-up). The mean followup time for HIV-positive and -negative patients was $14 \cdot 1 \pm 8 \cdot 73$ months and $17 \cdot 8 \pm 7 \cdot 80$ months,

Table 4 Basis of diagnosis of TB

\begin{tabular}{|c|c|c|c|}
\hline Basis of diagnosis $n$ (\%) & $\begin{array}{c}\text { HIV }+ \\
(n=150)\end{array}$ & $\begin{array}{c}\text { HIV- } \\
(n=155)\end{array}$ & $\begin{array}{c}\text { Overall } \\
(n=305)\end{array}$ \\
\hline Smear/culture $^{+}$ & $70(46.7)$ & $120(77.4)$ & $190(62.3)$ \\
\hline $\mathrm{PCR}^{+}$ & $4(2.7)$ & $1(0.6)$ & $5(1.6)$ \\
\hline Cyto-/Histopathology & $11(7.3)$ & $15(9.7)$ & $26(8.5)$ \\
\hline $\begin{array}{l}\text { Chemistry (raised fluid } \\
\text { ADA levels) }\end{array}$ & 0 & $1(0.6)$ & $1(0.3)$ \\
\hline Radio-clinical & $65(43.3)$ & $18(11.6)$ & $83(27.2)$ \\
\hline
\end{tabular}

${ }^{+}$Constitute definitive diagnosis.

N.B. in case of more than one diagnostic criterion being suggestive of TB, that with highest value was taken, in the order: smear/culture $>$ PCR $>$ cyto-/ histopathology > chemistry > radio-clinical. 
Table 5 Treatment outcome at end of initial ATT

\begin{tabular}{lccc}
\hline $\begin{array}{l}\text { TREATMENT OUTCOME } \\
\text { number (percent) }\end{array}$ & $\begin{array}{c}\text { HIV }+ \\
(\mathbf{n}=\mathbf{1 5 0 )}\end{array}$ & $\begin{array}{c}\text { HIV- } \\
(\mathbf{n}=\mathbf{1 5 5 )}\end{array}$ & P-value \\
\hline Treatment success & \\
Treatment completed & $\mathbf{1 1 5 ( 7 6 . 7 )}$ & $\mathbf{1 4 5}(93.5)$ & $<\mathbf{0 . 0 0 1}$ \\
Cure & $85(56.7)$ & $45(29.0)$ & \\
Treatment failure & $30(20.0)$ & $100(64.5)$ & \\
Default & $\mathbf{8 ( 5 . 3 )}$ & $\mathbf{4 ( 2 . 6 )}$ & $\mathbf{0 . 1 6}$ \\
Lost to follow-up & $4(2.7)$ & 0 & \\
Death & $9(6.0)$ & $4(2.6)$ & \\
Treatment modified & $\mathbf{1 3 ( 8 . 7 )}$ & $\mathbf{2 ( 1 . 3 )}$ & $<\mathbf{0 . 0 1}$ \\
\hline
\end{tabular}

+ Sum of cure and treatment completed.

respectively. Attrition rates were $24.5 \%$ in the HIVpositive group, and $19.3 \%$ of HIV-negative patients, despite attempts (telephone, home visits) to find those who were lost to follow-up.

Uni- and multivariate analysis of independent variables found no predictor of unfavourable outcome (i.e. any outcome other than treatment success) in the two groups. Variables included various blood test results at baseline (erythrocyte sedimentation rate, serum albumin, haemoglobin, CD4 cell count), chest X-ray severity, sex, age, presence of dissemination of TB, body mass index, and whether the $\mathrm{TB}$ was pulmonary or extrapulmonary.

The median time of relapse after treatment was six months. Relapse rate in percentage per person year observed (PYO) was calculated: in HIV-positive patients, $3 \cdot 48 \%$ PYO (95\% confidence interval [CI]: 0 - 43-6.54\%), and $0 \cdot 91 \%$ PYO (CI: $0 \cdot 35-2 \cdot 18 \%)$ in HIV-negative patients $[p=0 \cdot 09]$. Of the five HIV-positive relapses, three were pulmonary; the two HIV-negative patients both suffered pulmonary relapses. Of the four HIV-positive pulmonary TB patients, 3 had different spoligotyping genotypes suggesting exogenous re-infection while one had similar genotype suggesting endogenous reactivation. One patient in the HIV-negative group had similar genotype.

Table 6 Changes in various parameters between baseline and 6 months of treatment

\begin{tabular}{|c|c|c|c|c|}
\hline \multirow[b]{2}{*}{ Parameter } & \multicolumn{2}{|c|}{ HIV+ } & \multicolumn{2}{|c|}{ HIV- } \\
\hline & Pre & Post & Pre & Post \\
\hline BMI, mean, $\mathrm{kg} / \mathrm{m}^{2}$ & 18.1 & 19.8 & 17.9 & 19.7 \\
\hline Haemoglobin, mean, g/dl & 10.0 & 12.3 & 11.1 & 13.2 \\
\hline ESR, mean, mm/h & 49.4 & 34.1 & 43.7 & 24.2 \\
\hline Albumin, mean, g/dl & 3.5 & 4.3 & 4.2 & 4.7 \\
\hline CD4 cell count, mean, cells $/ \mathrm{mm}^{3}$ & 194.1 & 330.8 & 593.2 & 676.5 \\
\hline HIV viral load, median, copies/ml & 170339 & 400 & - & - \\
\hline
\end{tabular}

Bold type = statistically significant difference in degree of change between the two groups $(p<0.05)$.
Table 7 Treatment outcome in smear-positive pulmonary TB patients at end of initial ATT

\begin{tabular}{lccc}
\hline $\begin{array}{l}\text { TREATMENT OUTCOME } \\
\text { number (percent) }\end{array}$ & HIV + ( $\mathbf{n}=\mathbf{5 2})$ & HIV- $(\mathbf{n}=\mathbf{1 0 5 )}$ & P-value \\
\hline Treatment success $^{+}$ & $\mathbf{3 6}(\mathbf{6 9 . 2 )}$ & $\mathbf{1 0 0}(\mathbf{9 5 . 2 )}$ & $<\mathbf{0 . 0 0 1}$ \\
Treatment completed $^{\wedge}$ & $6(11.5)$ & $5(4.8)$ & \\
Cure & $30(57.7)$ & $95(90.5)$ & \\
Treatment failure & $2(3.8)$ & $2(1.9)$ & \\
Lost to follow-up & $8(15.4)$ & $2(1.9)$ & \\
Death & $6(11.5)$ & $1(1.0)$ &
\end{tabular}

+ Sum of cure and treatment completed.

$\wedge$ These patients were either diagnosed by bronchoscopy and smear positive aspirate, or unable to produce sputum to provide evidence of sputum conversion and therefore cure, but had clinical and radiological resolution at end of treatment.

\section{HIV-specific outcomes}

Of the HIV-positive patients, all but one were started on highly active ART (one died before commencement). The median interval between starting the ATT and ART was 30 days (interquartile range: 17-60 days). Zidovudinebased therapy was given to $65 \%$, the remaining $35 \%$ (53/150) received stavudine.

Mean rise in CD4 cell count and median fall in log plasma HIV viral load over the TB treatment phase were statistically significant $(\mathrm{P}<0.001)$.

Co-trimoxazole prophylaxis was given in 134/150 $(89 \cdot 3 \%)$. Fourteen patients were found to have opportunistic infections other than TB, some with multiple infections. The majority of these were present at baseline assessment.

Table 8 Treatment outcome at $\mathbf{2 4}$ months follow-up*

\begin{tabular}{|c|c|c|c|}
\hline $\begin{array}{l}\text { TREATMENT OUTCOME } \\
\text { number (percent) }\end{array}$ & $\begin{array}{c}\text { HIV + } \\
(n=102)\end{array}$ & $\begin{array}{c}\text { HIV- } \\
(n=109)\end{array}$ & P-value \\
\hline Treatment success $^{+}$ & $41(40.2)$ & $79(72.5)$ & $P<0.001$ \\
\hline Treatment completed & 37 (36.3) & $38(34.9)$ & \\
\hline Cure & $4(3.9)$ & $41(37.6)$ & \\
\hline $\begin{array}{l}\text { Treatment success } \\
\text { [excluding those lost to } \\
\text { follow up and defaulters]^ }\end{array}$ & $41(53.2)$ & $79(89.8)$ & $P<0.001$ \\
\hline Treatment failure & $8(7.8)$ & $4(3.7)$ & \\
\hline Relapse & $5(4.9)$ & $2(1.8)$ & \\
\hline Default & $4(3.9)$ & 0 & \\
\hline Lost to follow-up & $21(20.6)$ & $21(19.3)$ & \\
\hline Death & $22(21.6)$ & $3(2.8)$ & $P<0.001$ \\
\hline Treatment modified & $1(1.0)$ & 0 & \\
\hline \multicolumn{4}{|c|}{$\begin{array}{l}\text { * Includes patients completing } 24 \text { months follow-up and those being labelled } \\
\text { as death, lost to follow-up, failure and relapse - i.e. does not include patients } \\
\text { still under active follow-up. } \\
+ \text { Sum of cure and treatment completed. } \\
\wedge \text { In this outcome, the 'default' and 'lost to follow up' groups have been } \\
\text { removed from the cohort, so that for HIV }+n=77 \text {, and for HIV- } n=88 \text {. }\end{array}$} \\
\hline
\end{tabular}


Table 9 Adverse events in each group while on ATT

\begin{tabular}{lccc}
\hline & $\begin{array}{c}\text { HIV }+ \\
(\mathbf{n}=\mathbf{1 5 0})\end{array}$ & $\begin{array}{c}\text { HIV- } \\
(\mathbf{n}=\mathbf{1 5 5})\end{array}$ & $\begin{array}{c}\text { Overall } \\
(\mathbf{n = 3 0 5})\end{array}$ \\
\hline Total patients experiencing adverse & 69 & 74 & 143 \\
event(s) & $(46.0 \%)$ & $(47.7 \%)$ & $(46.9 \%)$ \\
Total patients experiencing & 44 & $3(1.9 \%)$ & 47 \\
serious adverse event(s) & $(29.3 \%)$ & & $(15.4 \%)$ \\
IRIS* & $14(9.3 \%)$ & 0 & $14(4.6 \%)$ \\
Anaemia (new on treatment)* & $9(6.0 \%)$ & 0 & $9(3.0 \%)$ \\
Drug-induced hepatotoxicity* & $5(3.3 \%)$ & 0 & $5(1.6 \%)$ \\
Isoniazid-induced psychosis* & $1(0.7 \%)$ & 0 & $1(0.3 \%)$ \\
Stevens-Johnson syndrome* & $1(0.7 \%)$ & 0 & $1(0.3 \%)$ \\
Peripheral neuropathy* & $2(1.3 \%)$ & 0 & $2(0.7 \%)$ \\
Haemoptysis* & 0 & $6(3.9 \%)$ & 0 \\
Itch & $9(6.0 \%)$ & $2(1.3 \%)$ & $11(3.6 \%)$ \\
Rash & $11(7.3 \%)$ & $2(1.3 \%)$ & $13(4.3 \%)$ \\
Nausea/vomiting & 47 & 71 & 118 \\
& $(31.3 \%)$ & $(45.8 \%)$ & $(38.7 \%)$ \\
Diarrhoea & 17 & 24 & 41 \\
& $(11.3 \%)$ & $(15.5 \%)$ & $(13.4 \%)$ \\
Abdominal pain & 17 & 26 & 43 \\
& $(11.3 \%)$ & $(16.8 \%)$ & $(14.1 \%)$ \\
\hline
\end{tabular}

* Classified as serious adverse events.

Uni- and multivariate analysis of independent risk factors for mortality in the HIV-positive group found only baseline CD4 cell count to be significant. Patients with CD4 cell count of less than 100 cells $/ \mathrm{mm}^{3}$ had an odds ratio of 8.43 for mortality ( $\mathrm{p}=0 \cdot 013$; CI: $1 \cdot 58-44 \cdot 95)$.

\section{Adverse events}

Table 9 details adverse events in the two groups while on treatment (totals and selected events). A similar number of non-serious adverse events were encountered in both groups $(\mathrm{p}=0.851)$, while significantly more serious adverse events were observed in the HIV-positive patients $(\mathrm{p}<0.001)$. Anaemia prompted switch from zidovudine to stavudine, while drug induced hepatotoxicity was treated by temporary ATT modification. Besides deaths and IRIS (see below), no other serious adverse event was observed.

Immune reconstitution inflammatory syndrome (IRIS) occurred in 14 patients at a median of 76.5 days after commencing ART; all were mild and did not require discontinuation of treatment. There was a significant increase in median CD4 cell count $(\mathrm{p}=0.001)$ and decrease in mean log plasma viral load $(\mathrm{p}<0.0001)$ between preIRIS and post-IRIS values.

In total, $22 \mathrm{HIV}$-positive and $3 \mathrm{HIV}$-negative patients died. Of the HIV-positive deaths, 5 were within the first 2 months, a further 8 on treatment, and 9 were during follow-up; the median time of death was 5.5 months after enrolment. Four deaths (three HIV-positive, one
HIV-negative) were confirmed as due to TB. Insufficient details were available for the remaining patients to determine causality, as they died at home or at a different hospital.

\section{Discussion}

The proportion of HIV-negative patients achieving treatment success was much higher than HIV-positive patients: at the end of treatment, $93.5 \%$ versus $76.7 \%$ $(\mathrm{P}<0.001)$; at the end of treatment in those with sputum positive pulmonary TB, $95.2 \%$ versus $69.2 \%(\mathrm{P}<0.001)$; and at 24 months follow up excluding those lost to follow up, $89.8 \%$ versus $53.2 \%(\mathrm{P}<0.001)$. Multivariate analysis did not reveal any independent risk factor for not achieving treatment success within each group.

Higher treatment success in HIV-negative and higher mortality in HIV-positive patients in the present study are in agreement with previous studies $[8,13]$. Indeed, the end of treatment mortality rate in the present study's co-infected patients $(8 \cdot 1 \%)$ is lower than most previous figures - Khan et al's meta-analysis quotes 15\% [16]. In many studies, no patients received ART (unlike universal highly active ART in the present one), which may explain higher mortality. Abdool Karim et al. found in 2010 that ART during ATT reduced mortality by $56 \%$, to just $5.4 \%$ in median 12.1 months follow-up [2]. Interestingly, recent studies without concurrent ART quote $10 \cdot 4 \%$ [12] and $10 \cdot 9 \%$ [18], which suggests the fall in mortality may be due in part to other improvements in HIV/TB care.

Mortality was largely after the intensive phase of ATT, with half of the HIV positive patients dying in the follow-up stage, which has been suggested previously $[2,11,12]$. Only four deaths in the present study were attributable to TB. These observations may implicate HIV/AIDS as a more important factor for mortality than $\mathrm{TB}$, though paucity of death causation data means this assumption is difficult to make. However, lower nadir CD4 counts in patients who subsequently died support this hypothesis, which is often related to delayed presentation, described previously at our Centre [20].

Multivariate analysis revealed those with CD4 count $<100$ cells $/ \mathrm{mm}^{3}$ had 8 -fold higher risk of dying. This has been shown previously $[2,12]$, and suggests that immunological effects of HIV need close attention in TB-HIV coinfection, with early ART commencement being advocated by most bodies $[2,3]$. It may also mean that this subgroup may be considered for targeted interventions such as daily therapy, extended duration or otherwise.

Treatment failure and relapse rates were not significantly different in HIV-positive and HIV-negative patients. Previous publications are divided on these with regards to HIV status [5]. Most studies have shown similar failure rates regardless of HIV status [13,27], which is 
observed in the present study. Some studies report relapse being more common in HIV co-infection [11,28]. Pooled data show a higher relapse rate (12\% PYO) in HIV-positive individuals than reported in this study (3. 48\% PYO) [16]. Spoligotyping in HIV-positive patients revealed three out of four having exogenous reinfection with $\mathrm{TB}$ - reports of the proportion being reinfected vary, however, the number of relapses are too small in this case to make any conclusions. This may reflect inadequate airborne infection control measures.

Sputum smear and culture conversion rates at 2 months were found to be similar regardless of HIV status. The 2-month culture conversion rate for HIV-positive individuals $(85 \cdot 3 \%)$ was in accordance with earlier reports, with a range of $74-95 \%$ being quoted $[11,17,18]$.

Extension of ATT of 1-3 months was required in 35 of the HIV-positive patients, but only 13 in the HIVnegative group (median 1 month). A trial comparing 6 with 9 months thrice weekly ATT demonstrated lower bacteriological recurrence with 9 months treatment, but found no other benefits [12]. Hence it is unclear whether longer treatment would be beneficial.

HIV-infected patients were started on ART a median of 30 days after ATT, with good immunological and virological response. This is representative of present care of such patients according to recent WHO guidance stating all people with HIV-TB co-infection should be started on ART early in the ATT course [3]. It correlates well with other studies looking at outcomes of HIV-TB co-infection with early/concurrent ART [2,29].

With regards to safety, a similar frequency of nonserious adverse events was found in each group; though this seems higher than in several studies, reporting up to $37 \%$ of patients experiencing any event $[12,13,17]$. Such events were all self-limiting or easily treated, and may reflect the close monitoring in this study. The significantly higher incidence of serious adverse events in the HIV-positive group was mostly made up of deaths and HIV-specific events. The $29.3 \%$ figure observed is similar to that observed in the 'integrated therapy' group of Abdool Karim et al's recent study [2]. In addition, findings of the present study are similar to previous reports of low hepatotoxicity figures with thrice weekly treatment regimens [30].

The study depicts an up-to-date comparison of outcomes of thrice weekly six-month ATT in HIV-positive and negative individuals. Strengths include: supplementation of currently lacking follow-up data on outcomes of intermittent ATT in HIV co-infected patients, especially in terms of relapse rates and late mortality; the real-life situation has been studied, with inclusion of smear-negative and extrapulmonary patients, concurrent ART, and preservation of the national TB programme's treatment protocol; and surveillance results, including sputum culture conversion rate and post-ATT follow-up, in Indian patients (currently absent from national programme reports).

Limitations of this study include: 1) Lack of routine DST in order to screen for drug resistance: this is not universally recommended for patients with a new TB diagnosis by Indian or WHO guidelines [3,24], but is increasingly being used in research settings [5,12]. 2) The high attrition rate, especially in the HIV-positive group and after initial treatment, may have impacted on completeness of follow-up data. This is a common problem in resource-limited settings, as highlighted previously at this institute's ART clinic [20]. 3) A significant proportion of patients were not definitive (bacteriologically confirmed) cases of $\mathrm{TB}$, despite thorough investigation to exclude other diagnoses and most having response to treatment. 4) The study was observational, with resulting lack of control of confounding factors, evidenced by baseline heterogeneity. 5) As numbers of relapse and failure cases were small, it is difficult to judge differences between the two groups with confidence in this respect.

\section{Conclusions}

Intermittent ATT is desirable in developing country settings to maintain direct observation with minimal strain on stretched health systems. However it has been poorly studied [31]. The present study shows excellent outcomes and safety of thrice-weekly ATT in HIV-negative individuals, confirming its adequacy in these patients [3], though acquired rifampicin resistance may be promoted, which needs detailed study $[3,5,12]$. Outcomes in HIV co-infection do not seem to be as good, with lower treatment success and higher mortality being observed, though with adequate safety. This should prompt further focussed study on treatment options for these patients, such as a trial starting at this institute examining outcomes of daily versus thrice-weekly therapy. National programme coordinators should re-visit guidelines, and investigate whether therapy changes in HIV co-infection should be universal, as recommended by the WHO [3], or in specific subgroups, such as those with low CD4 counts, to balance attempts to reduce morbidity and mortality in TB-HIV co-infection with awareness of resource constraints.

\section{Abbreviations}

ART: Anti-retroviral therapy; ATT: Anti-tuberculosis therapy; Cl: Confidence interval; DOTS: Directly observed treatment, short course; DST: Drug susceptibility testing; ELISA: Enzyme-linked immunosorbent assay; HIV: Human immunodeficiency virus; IRIS: Immune reconstitution inflammatory syndrome; MDR: Multi-drug resistant; PCR: Polymerase chain reaction; PYO: Person year(s) observed; TB: Tuberculosis; WHO: World Health Organization.

\section{Competing interests}

All authors: no competing interests. 


\section{Authors' contributions}

SKS designed the study. SKS, KM, SD, SR, BS, RV, DG and SS collected data. SKS, KM, BS and VS analysed the data and contributed to the first draft of the manuscript. All authors finalised, read and approved the final manuscript.

\section{Acknowledgements}

We thank the administrative, research, laboratory and clinic staff in the Department of Medicine, All India Institute of Medical Sciences. This work was funded by the National AIDS Control Organisation, Ministry of Health and Family Welfare, Government of India, New Delhi, India. The sponsoring organisation played no part in study design, data collection or analysis, or writing or submission of the report.

\section{Author details}

'Department of Medicine, All India Institute of Medical Sciences, Ansari Nagar, New Delhi 110029, India. ${ }^{2}$ Department of Biostatistics, All India Institute of Medical Sciences, Ansari Nagar, New Delhi 110029, India.

Received: 8 February 2013 Accepted: 2 September 2013 Published: 7 October 2013

\section{References}

1. Corbett EL, Watt CJ, Walker N, Maher D, Williams BG, Raviglione MC, Dye C The growing burden of tuberculosis: global trends and interactions with the HIV epidemic. Arch Intern Med 2003, 163:1009-1021.

2. Abdool Karim SS, Naidoo K, Grobler A, Padayatchi N, Baxter C, Gray A, Gengiah T, Nair G, Bamber S, Singh A, Khan M, Pienaar J, El-Sadr W, Friedland $G$, Abdool KQ: Timing of initiation of antiretroviral drugs during tuberculosis therapy. N Engl J Med 2010, 362:697-706.

3. World Health Organization (WHO): Treatment of tuberculosis: Guidelines. 4th ed. Geneva: WHO Press; 2010.

4. De Jong BC, Israelski DM, Corbett EL, Small PM: Clinical management of tuberculosis in the context of HIV infection. Annual Rev Med 2004, 55:283-301.

5. Perlman DC, Leung CC, Yew WW: Treatment of tuberculosis in HIV-infected patients: we need to know more. Am J Respir Crit Care Med 2007, 175(11):1102-1103.

6. American Thoracic Society (ATS), Centers for Disease Control and Prevention (CDC), Infectious Diseases Society of America (IDSA): Treatment of tuberculosis. Am J Respir Crit Care Med 2003, 167:603-662

7. Dlodlo RA, Fujiwara PI, Enarson DA: Should tuberculosis treatment and control be addressed differently in HIV-infected and -uninfected individuals? Eur Respir J 2005, 25:751-757

8. Sterling TR, Alwood K, Gachuhi R, Coggin W, Blazes D, Bishai WR, Chaisson RE: Relapse rates after short-course (6-month) treatment of tuberculosis in HIV-infected and uninfected persons. AIDS 1999, 13:1899-1904.

9. Chaisson RE, Clermont HC, Holt EA, Cantave M, Johnson MP, Atkinson J, Davis H, Boulos R, Quinn TC, Halsey NA: Six-month supervised intermittent tuberculosis therapy in Haitian patients with and without HIV infection. Am J Respir Crit Care Med 1996, 154(4):1034-1038.

10. Kassim S, Sassan-Morokro M, Ackah A, Abouya LY, Digbeu H, Yesso G, Coulibaly IM, Coulibaly D, Whitaker PJ, Doorly R, Vetter KM, Brattegaard K, Gnaore E, Greenberg AE, Wiktor SZ, De Cock KM: Two-year follow-up of persons with HIV-1 and HIV-2-associated pulmonary tuberculosis treated with short-course chemotherapy in West Africa. AIDS 1995, 9(10):1185-1192.

11. Nahid P, Gonzalez LC, Rudoy I: Treatment outcomes of patients with HIV and tuberculosis. Am J Respir Crit Care Med 2007, 175(11):1199-1206.

12. Swaminathan $S$, Narendran G, Venkatesan P, Illiayas S, Santhanakrishnan R, Menon PA, Padmapriyadarsini C, Ramachandran R, Chinnaiyan P, Suhadev M, Sakthivel R, Narayanan PR: Efficacy of a 6-month versus 9-month intermittent treatment regimen in HIV-infected patients with tuberculosis: randomized clinical trial. Am J Respir Crit Care Med 2010, 181:743-751.

13. Perriens JH, St Louis ME, Mukadi YB, Brown C, Prignot J, Pouthier F, Portaels F, Willame JC, Mandala JK, Kaboto M, Ryder RW, Roscigno G, Piot P: Pulmonary tuberculosis in HIV-infected patients in Zaire: a controlled trial of treatment for either 6 or 12 months. N Engl J Med 1995, 332:779-784.

14. El Sadr WM, Perlman DC, Matts JP, Nelson ET, Cohn DL, Salomon N, Olibrice M, Medard F, Chirgwin KD, Mildvan D, Jones BE, Telzak EE, Klein O, Heifets L, Hafner R: Evaluation of an intensive intermittent-induction regimen and duration of short-course treatment for human immunodeficiency virus-related pulmonary tuberculosis. Clin Infect Dis 1998, 26(5):1148-1158.
15. Jindani A, Nunn AJ, Enarson DA: Two 8-month regimens of chemotherapy for treatment of newly diagnosed pulmonary tuberculosis: international multicentre randomised trial. Lancet 2004, 364:1244-1251.

16. Khan FA, Minion J, Pai M, Royce S, Burman W, Harries AD, Menzies D: Treatment of active tuberculosis in HIV-coinfected patients: a systematic review and meta-analysis. Clin Infect Dis 2010, 50(9):1288-1299.

17. Johnson JL, Okwera A, Nsubuga P, Nakibali JG, Whalen CC, Hom D, Cave MD, Yang ZH, Mugerwa RD, Ellner JJ: Efficacy of an unsupervised 8-month rifampicin-containing regimen for the treatment of pulmonary tuberculosis in HIV-infected adults. Uganda-Case Western Reserve University Research Collaboration. Int J Tuberc Lung Dis 2000, 4(11):1032-1040.

18. Okwera A, Johnson JL, Luzze H, Nsubuga P, Kayanja H, Cohn DL, Nunn P, Ellner JJ, Whalen CC, Mugerwa RD: Comparison of intermittent ethambutol with rifampicin-based regimens in HIV-infected adults with PTB, Kampala. Int J Tuberc Lung Dis 2006, 10(1):39-44.

19. Schwander S, Rusch-Gerdes S, Mateega A, Lutalo T, Tugume S, Kityo C, Rubaramira R, Mugyenyi P, Okwera A, Mugerwa R: A pilot study of antituberculosis combinations comparing rifabutin with rifampicin in the treatment of HIV-1 associated tuberculosis. A single-blind randomized evaluation in Ugandan patients with HIV-1 infection and pulmonary tuberculosis. Tuber Lung Dis 1995, 76(3):210-218.

20. Sharma SK, Dhooria S, Prasad KT, George N, Ranjan S, Gupta D, Sreenivas V, Kadhiravan T, Miglani S, Sinha S, Wig N, Biswas A, Vajpayee M: Outcomes of antiretroviral therapy in a northern Indian urban clinic. Bull World Health Organ 2010, 88:222-226.

21. Karmakar S, Sharma SK, Vashishta R, Sharma A, Ranjan S, Gupta D, Sreenivas V, Sinha S, Biswas A, Gulati V: Clinical characteristics of tuberculosisassociated immune reconstitution inflammatory syndrome in North Indian population of HIV/AIDS patients receiving HAART. Clin Dev Immunol 2011, 2011:239021.

22. National Tuberculosis Association of the USA: Diagnostic standards and classification of tuberculosis. New York: National Tuberculosis Association; 1961.

23. World Health Organization: Laboratory services in tuberculosis control II. Microscopy. Italy: WHO Press; 1999.

24. Central TB Division, Directorate General of Health Services, Ministry of Health and Family Welfare: Tuberculosis control in India. New Delhi: Elsevier; 2005

25. National AIDS Control Organisation (NACO): Antiretroviral therapy guidelines for HIV-infected adults and adolescents including post-exposure prophylaxis. New Delhi: NACO; 2007.

26. Kamerbeek J, Schouls L, Kolk A, van Agterveld M, van Soolingen D, Kuijper S,

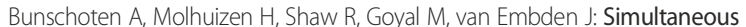
detection and strain differentiation of Mycobacterium tuberculosis for diagnosis and epidemiology. J Clin Microbiol 1997, 35:907-914

27. Daniel OJ, Alausa OK: Treatment outcome of TB/HIV positive and TB/HIV negative patients on directly observed treatment, short course (DOTS) in Sagamu. Nigeria. Niger J Med 2006, 15(3):222-226.

28. Korenromp EL, Scano F, Williams BG, Dye C, Nunn P: Effects of human immunodeficiency virus infection on recurrence of tuberculosis after rifampin-based treatment: an analytical review. Clin Infect Dis 2003, 37:101-112

29. Blanc FX, Sok T, Laureillard D, Borand L, Rekacewicz C, Nerrienet E, Madec Y, Marcy O, Chan S, Prak N, Kim C, Lak KK, Hak C, Dim B, Sin Cl, Sun S, Guillard B, Sar B, Vong S, Fernandez M, Fox L, Delfraissy JF, Goldfeld AE, CAMELIA (ANRS 1295-CIPRA KH001) Study Team: Earlier versus later start of antiretroviral therapy in HIV-infected adults with tuberculosis. N Engl J Med 2011, 365(16):1471-1481

30. Dhingra VK, Rajpal S, Aggarwal N, Aggarwal JK, Shadab K, Jain SK: Adverse drug reactions observed during DOTS. J Commun Dis 2004, 236:251.

31. Mwandumba HC, Squire SB: Fully intermittent dosing with drugs for treating tuberculosis in adults. Cochrane Database Syst Rev 2001, 4:CD000970

doi:10.1186/1471-2334-13-468

Cite this article as: Vashishtha et al.: Efficacy and safety of thrice weekly DOTS in tuberculosis patients with and without HIV co-infection: an observational study. BMC Infectious Diseases 2013 13:468. 